\title{
EPIDEMICS ON RANDOM GRAPHS WITH TUNABLE CLUSTERING
}

\author{
TOM BRITTON, ${ }^{* * *}$ \\ MARIA DEIJFEN, ${ }^{* * * *}$ \\ ANDREAS N. LAGERÅS***** AND \\ MATHIAS LINDHOLM, ${ }^{* * * * * *}$ Stockholm University
}

\begin{abstract}
In this paper a branching process approximation for the spread of a Reed-Frost epidemic on a network with tunable clustering is derived. The approximation gives rise to expressions for the epidemic threshold and the probability of a large outbreak in the epidemic. We investigate how these quantities vary with the clustering in the graph and find that, as the clustering increases, the epidemic threshold decreases. The network is modeled by a random intersection graph, in which individuals are independently members of a number of groups and two individuals are linked to each other if and only if there is at least one group that they are both members of.
\end{abstract}

Keywords: Epidemics; random graph; clustering; branching process; epidemic threshold

2000 Mathematics Subject Classification: Primary 92D30; 05C80

\section{Introduction}

This paper is concerned with Reed-Frost epidemics modified to take place on random networks. Introduced in 1928 by two medical researchers, Lowell Reed and Wade Frost, the Reed-Frost model is one of the simplest stochastic epidemic models. The spread of the infection takes place in generations: each individual that is infective at time $t(t=0,1, \ldots)$ independently makes contacts with all other individuals in the population with some probability $p$, and if a contacted individual is susceptible, it becomes infected at time $t+1$. Also, at time $t+1$, the infective individuals from time $t$ are removed from the epidemic process.

The behavior of the Reed-Frost model is well understood; see, e.g. von Bahr and Martin-Löf (1980). A crucial assumption which simplifies the analysis of the model is that the population in which the epidemic takes place is taken to be homogeneously mixing, that is, an infective individual is assumed to make contacts with all other individuals in the population with the same probability. This assumption is of course very unrealistic, since, in a real-life epidemic, an infective individual is much more likely to infect individuals with whom he/she has some kind of social connection. The Reed-Frost model can easily be adapted to incorporate this type of heterogeneity by introducing a graph to represent the social structure in the population and then stipulating that infective individuals can only infect their neighbors in the social network;

Received 30 July 2007; revision received 19 May 2008.

* Postal address: Department of Mathematics, Stockholm University, 10691 Stockholm, Sweden.

** Email address: tomb@math.su.se

*** Email address: mia@math.su.se

**** Email address: andreas@math.su.se

***** Email address: lindholm@math.su.se 
see Section 3. This modification makes the analysis of the model two-fold. Firstly, finding a realistic model for the underlying social network, and, secondly, studying the behavior of the epidemic on this graph.

Large complex networks such as social contact structures, the Internet, and various types of collaboration networks have received a lot of attention during the last few years; see, e.g. Dorogovtsev and Mendes (2003), Newman et al. (2006), and the references therein. As for social networks, one of their most striking features is that they are highly clustered, meaning roughly that there is a large number of triangles and other short cycles; see, e.g. Newman (2003b). This is a consequence of the fact that friendship circles are typically strongly overlapping, so that many of our friends are also friends of each other. A model that captures this in a natural way is the so-called random intersection graph, which is described in Section 2. Roughly, the idea of the model is that people are members of groups - families, schools, workplaces etc.—and an edge is drawn between two individuals if there is at least one group that they are both members of. If the relation between the number of individuals and the number of groups is chosen appropriately, this leads to a graph where the amount of clustering can be tuned by adjusting the parameters of the model.

An important goal of network modeling is to investigate how the structure of the network affects the behavior of various types of dynamic processes on the network; see Durrett (2006) for an overview. When it comes to epidemics, Andersson (1999) is a comprehensible introduction, in which expressions for the epidemic threshold, the probability of a large outbreak, and the final size of the epidemic are derived in a heuristic way for a number of underlying graphs. Here, the epidemic threshold, commonly denoted by $R_{0}$, is defined as a function of the parameters of the model such that a large outbreak in the epidemic has positive probability if and only if $R_{0}>1$. In epidemic modeling a common technique for deriving expressions for the epidemic threshold and the probability of a large outbreak is to use branching process approximations of the early stages of the epidemic. However, when studying epidemics on networks, dependencies between the edges in the graph tend to make branching process approximations more complicated. Results for epidemics on graphs with arbitrary degree distribution can be found in Andersson (1998), and Erdős-Rényi graphs and some extensions thereof are dealt with in Neal (2004), (2006). There is however very little work done on more complicated graph structures.

The aim of this paper is to give a rigorous analysis of how clustering in a network affects the spread of an epidemic. The network is modeled by a random intersection graph with tunable clustering and we then let a Reed-Frost epidemic propagate on this graph. Comparing the epidemic with a certain branching process yields (implicit) expressions for the epidemic threshold and the probability of a large outbreak. Numerical evaluations reveal that, as the clustering increases, the epidemic threshold decreases-that is, large outbreaks are possible for larger parts of the parameter space-but also that the actual value of the probability of a large outbreak decreases as the clustering approaches its maximal value. To the authors' knowledge, this is the first rigorous investigation of how the spread of an epidemic is affected by clustering.

In Newman (2003a), the effect of clustering on epidemics was studied by heuristic means, and calculations therein indicate that indeed the epidemic threshold should decrease as the clustering increases. Furthermore, Trapman (2007) studied epidemics on graphs with a given expected number of triangles, but the construction of the graph is more involved there. We also mention the work in Ball et al. (1997) on the so-called household model, which describes the spread of an epidemic in a population with group structure. The model there, however, is not formulated in terms of an underlying graph and the concept of clustering is not considered. 
The paper is organized as follows. In Section 2 random intersection graphs and their properties are described in more detail. Section 3 contains the main result-a comparison of a Reed-Frost epidemic on a random intersection graph with a branching process-and its proof. In Section 4 the final size of the epidemic is commented on. It is observed that a thinned random intersection graph is in fact not a random intersection graph, implying that results concerning the component structure in a random intersection graph cannot be used to draw conclusions about the final size of the epidemic. In Section 5 numerical results are presented and, finally, Section 6 contains a short discussion.

\section{Random intersection graphs}

Random intersection graphs were introduced in Singer (1995) and Karoński et al. (1999). In its simplest form, the model is defined as follows: given a set $\mathcal{V}$ of $n$ vertices and a set $\mathcal{A}$ of $m$ auxiliary vertices, construct a bipartite graph $\mathscr{B}_{n, m, r}$ by letting each edge between vertices $v \in \mathcal{V}$ and $a \in \mathcal{A}$ exist independently with probability $r$. The random intersection graph $g_{n, m, r}$ with vertex set $\mathcal{V}$ is obtained by connecting two vertices $v, w \in \mathcal{V}$ if and only if there is a vertex $a \in \mathcal{A}$ such that $a$ is linked to both $v$ and $w$ in $\mathscr{B}_{n, m, r}$. This construction can be generalized in various ways — see, e.g. Godehardt and Jaworski (2002) and Deijfen and Kets (2007)—but in this paper we will stick to the above formulation. We will also specialize to the case where $m=\left\lfloor\beta n^{\alpha}\right\rfloor$ for some constants $\alpha, \beta>0$ (where $\lfloor\cdot\rfloor$ denotes the integer part); see Karoński et al. (1999) for a motivation of this choice of $m$. In fact, to get a graph with tunable clustering, we will soon take $\alpha=1$.

If the vertices in $\mathcal{V}$ and $\mathcal{A}$ are thought of as individuals and groups, respectively, then the random intersection graph provides a model for a social network where individuals are connected if there is at least one group where they are both members. The probability that two individuals do not share any group is $\left(1-r^{2}\right)^{m}$, implying that the edge probability in the random intersection graph is $1-\left(1-r^{2}\right)^{m}$, and, hence, the expected degree of a fixed vertex is

$$
(n-1)\left(1-\left(1-r^{2}\right)^{m}\right)=\beta r^{2} n^{1+\alpha}+O\left(r^{4} n^{1+2 \alpha}\right) .
$$

To keep this expression bounded as $n \rightarrow \infty$, we let $r=\gamma n^{-(1+\alpha) / 2}$ for some $\gamma>0$. The expected degree then tends to $\beta \gamma^{2}$ as $n \rightarrow \infty$.

As for the asymptotic distribution of the vertex degree with the above choices of $m$ and $r$, it was shown in Stark (2004) to be a point mass at 0 for $\alpha<1$, a compound Poisson distribution, describing the law of a sum of a $\operatorname{Poisson}(\beta \gamma)$-distributed number of independent $\operatorname{Poisson}(\gamma)$ variables for $\alpha=1$, and a Poisson $\left(\beta \gamma^{2}\right)$ distribution for $\alpha>1$. To see this, note that the number of groups that an individual belongs to is binomially distributed with mean $m r=\beta \gamma n^{(\alpha-1) / 2}$. For $\alpha<1$, this goes to 0 as $n \rightarrow \infty$, explaining the point mass at 0 . For $\alpha=1$, the number of group memberships per individual is asymptotically Poisson $(\beta \gamma)$ distributed, and the sizes of the groups are Poisson $(\gamma)$, with overlaps between groups being very unlikely if $n$ is large, indicating that the degree distribution should indeed be compound Poisson. When $\alpha>1$, each individual belongs to infinitely many groups as $n \rightarrow \infty$. This means that the edge indicators are asymptotically independent, which suggests a Poisson distribution for the vertex degree. In fact, for $\alpha>1$, the random intersection graph is similar to the standard Erdős-Rényi random graph; see Fill et al. (2000).

Moving on to the clustering in the graph, for two vertices $v, w \in \mathcal{V}$, let $I_{v w}$ denote the edge indicator for the edge between $v$ and $w$ in $g_{n, m, r}$, and write $\mathrm{P}_{n}$ for the probability measure of 
$g_{n, m, r}$. We then define the clustering as

$$
c=c_{\alpha, \beta, \gamma}:=\lim _{n \rightarrow \infty} \mathrm{P}_{n}\left(I_{v w}=1 \mid I_{v u} I_{w u}=1\right),
$$

that is, $c$ is the limiting conditional probability that there is an edge between two vertices $v$ and $w$, given that they have a common neighbor $u$. The expected number of groups that individual $u$ belongs to is $\beta \gamma n^{(\alpha-1) / 2}$, which goes to $0, \beta \gamma$, or $\infty$ depending on whether $\alpha<1, \alpha=1$, or $\alpha>1$, respectively. As a consequence, the limiting probability that two individuals $v$ and $w$ who both share a group with $u$ in fact share the same group with $u$-thus being connected to each other-will behave differently depending on the value of $\alpha$. More specifically, it was shown in Deijfen and Kets (2007) that

$$
c_{\alpha, \beta, \gamma}= \begin{cases}1 & \text { if } \alpha<1 \\ (1+\beta \gamma)^{-1} & \text { if } \alpha=1 \\ 0 & \text { if } \alpha>1\end{cases}
$$

In view of the result in Stark (2004) concerning the degree distribution and the characterization of the clustering in Deijfen and Kets (2007), the best choice if we want to use a random intersection graph to describe a social network seems to be $\alpha=1$. This gives rise to a model where both the mean degree and the clustering can be tuned by adjusting the parameters $\beta$ and $\gamma$. More precisely, with $D$ denoting the limiting degree of a fixed vertex, we have

$$
\mathrm{E}[D]=\beta \gamma^{2} \quad \text { and } \quad c=(1+\beta \gamma)^{-1} \text {. }
$$

For the remainder of this paper, we fix $\alpha=1$ and write $g_{\beta, \gamma}^{(n)}=g^{(n)}$ and $\mathcal{B}_{\beta, \gamma}^{(n)}=\mathcal{B}^{(n)}$ for the corresponding random intersection graph and its underlying bipartite graph (omitting the subscripts when the dependence on $\beta$ and $\gamma$ does not need to be emphasized).

\section{The epidemic model and an approximating branching process}

Consider a closed homogeneous population consisting of $n$ individuals, labeled $v_{1}, \ldots, v_{n}$, with a social structure represented by a random intersection graph $g^{(n)}$. We will use the ReedFrost dynamics to describe the spread of an infection in this population. The social graph $g^{(n)}$ is assumed to be fixed throughout the spread of the infection. Furthermore, for simplicity, we start with one single randomly selected infective individual at time 0 , the rest of the population being susceptible. Without loss of generality, we assume that the initial infective, which will be referred to as the index case, is individual $v_{1}$. An individual that is infective at time $t$ $(t=0,1, \ldots)$ contacts each one of its neighbors in $g^{(n)}$ independently with some probability $p$, and if a contacted neighbor is susceptible, it becomes infective at time $t+1$. The individuals that were infective at time $t$ are removed from the epidemic process at time $t+1$ (by immunity or death) and take no further part in the spread of the infection.

We will be concerned with the set $\mathcal{E}^{(n)}$ of individuals that are ultimately affected by the above epidemic. More precisely, we will construct a branching process that can be used to determine whether $\mathcal{E}^{(n)}$ is finite or infinite in the limit as $n \rightarrow \infty$. To this end, first note that $\mathcal{E}^{(n)}$ can be identified with the cluster containing the index case in an edge percolation process on $g(n)$ in which each edge is open independently with probability $p$. Open edges in the percolation process are interpreted as possible transmission links for the disease, that is, if one of the vertices of an edge is infective at time $t$ and the other one is not, then the uninfected vertex becomes infective at time $t+1$. Furthermore, if we consider the percolation cluster of a particular vertex 
restricted to a subgraph of $g^{(n)}$ then the size of this cluster has the same distribution as the final size of a Reed-Frost epidemic on the subgraph. In particular, let $R_{k}(k=0, \ldots, n-1)$ be the size of the percolation cluster of a given vertex belonging to a complete subgraph with $k$ additional nodes, excluding the vertex itself. Then the distribution of $R_{k}$, denoted by $F_{k}$, is equivalent to the final size distribution of a Reed-Frost epidemic initiated by a single infectious individual in a homogeneously mixing population with $k$ susceptible individuals (excluding the index case). The distribution $F_{k}$ can be computed recursively; see Andersson and Britton (2000, Section 1.2).

We now define the branching process that will be used to approximate the epidemic process. To begin with, note that the groups in a random intersection graph generate complete subgraphs. Moreover, for a given individual who is a member of a given group, the additional number of group members in that group is $\operatorname{binomial}(n-1, \gamma / n)$ and, hence, asymptotically $\operatorname{Poisson}(\gamma)$. Thus, in the limit as $n \rightarrow \infty$, an individual will generate $R$ additional infected individuals in any group it is a member of, where

$$
R \sim F:=\sum_{k=0}^{\infty} F_{k} \frac{\gamma^{k}}{k !} \mathrm{e}^{-\gamma} .
$$

Recall that the number of groups that a given individual is a member of is asymptotically $\operatorname{Poisson}(\beta \gamma)$ distributed. Let $f$ be the generating function of a sum of a Poisson $(\beta \gamma)$ number of independent and identically distributed variables all distributed as $R$, that is,

$$
f(s)=\exp \left(\beta \gamma\left(\mathrm{E}\left[s^{R}\right]-1\right)\right),
$$

and let $\{Z(t): t \geq 0\}$ be a discrete-time branching process starting with a single individual $(Z(0)=1)$ and with offspring generating function $f$, that is, $\mathrm{E}\left[s^{Z(1)}\right]=f(s)$. Finally, write $E$ for the total progeny in such a process, that is,

$$
E=\sum_{t=0}^{\infty} Z(t)
$$

Let $E^{(n)}=\left|\mathcal{E}^{(n)}\right|$ denote the final size of a Reed-Frost epidemic on a random intersection graph $g^{(n)}$. Our main result is the following theorem, which will be proved by relating the initial phases of the epidemic to a branching process with the same distribution as $\{Z(t): t \geq 0\}$ as $n \rightarrow \infty$.

Theorem 3.1. As $n \rightarrow \infty$, we have $E^{(n)} \rightarrow E$ in distribution.

Define $\rho$ to be the smallest nonnegative root of the equation $f(\rho)=\rho$. It follows from standard results in branching processes theory that $\mathrm{P}(E=\infty)=1-\rho$ and that $\rho<1$ if and only if $E[Z(1)]>1$; see, e.g. Athreya and Ney (1972, Theorem 1). Combining this with Theorem 3.1 gives the following corollary concerning the asymptotic behavior of the epidemic.

Corollary 3.1. Define $R_{0}:=\mathrm{E}[Z(1)]=\beta \gamma \mathrm{E}[R]$, and write $\pi=1-\rho$. As $n \rightarrow \infty$, we have

(a) $E^{(n)} \rightarrow \infty$ with probability $\pi$,

(b) $\pi>0$ if and only if $R_{0}>1$. 
Before we continue with the proof of the theorem, we will state and prove a lemma concerning the bipartite graph $\mathscr{B}^{(n)}$. To this end, for an arbitrary graph $g$ with vertex set $\mathcal{W}$, the subgraph of $g$ induced by some subset $\mathcal{W}^{\prime} \subset \mathcal{W}$ is defined to be the subgraph consisting the vertices in $\mathcal{W}^{\prime}$ together with all the edges in $g$ that run between the vertices in $\mathcal{W}^{\prime}$. Let $\mathcal{C}^{(n)}(t)$ be the vertices of $\mathscr{B}^{(n)}$ at distance $t$ from vertex $v_{1}$. Note that a vertex in $\mathscr{B}^{(n)}$ may be either an individual (that is, a vertex $v \in \mathcal{V}$ ) or a group (that is, an auxiliary vertex $a \in \mathcal{A}$ ), and that vertices at an odd distance from $v_{1}$ correspond to groups and vertices at an even distance correspond to individuals.

Let $T_{n}$ be the event that the subgraph of $\mathscr{B}^{(n)}$ induced by $\mathcal{C}^{(n)}(\lfloor\kappa \log n\rfloor)$ is a tree.

Lemma 3.1. Let $\kappa>0$ be such that $1 / \kappa>2 \log \left(\beta \gamma^{2}\right)$. Then $\mathrm{P}\left(T_{n}\right) \rightarrow 1$ as $n \rightarrow \infty$.

Proof. We will build up $\mathcal{C}^{(n)}(t)$ by a sequence $\left\{\mathscr{D}^{(n)}(t): t \geq 0\right\}$, constructed in such a way that $\mathcal{C}^{(n)}(t)=\bigcup_{0 \leq s \leq t} \mathscr{D}^{(n)}(s)$. For odd $t$, the set $\mathscr{D}^{(n)}(t)$ will consist of groups and, for even $t$, the set $\mathscr{D}^{(n)}(t)$ will consist of individuals. To begin with, by definition, we have $\mathcal{C}^{(n)}(0)=\left\{v_{1}\right\}$, so necessarily $\mathscr{D}^{(n)}(0)=\mathcal{C}^{(n)}(0)$. For odd $t$, the set $\mathscr{D}^{(n)}(t)$ is then constructed by choosing, independently for each individual in $\mathscr{D}^{(n)}(t-1)$, a binomial $(m, \gamma / n)$-distributed number of distinct groups in $\mathcal{A}$, and, likewise, for even $t$, we construct $\mathscr{D}^{(n)}(t)$ by choosing, independently for each group in $\mathscr{D}^{(n)}(t-1)$, a binomial $(n, \gamma / n)$-distributed number of distinct individuals in $\mathcal{V}$. Let $X^{(n)}$ be a compound binomial random variable with generating function

$$
g(s)=\mathrm{E}\left[s^{X^{(n)}}\right]=\left(1-\frac{\gamma}{n}+\frac{\gamma}{n}\left(1-\frac{\gamma}{n}+\frac{\gamma}{n} s\right)^{n}\right)^{m}
$$

and let $\left\{X^{(n)}(t): t \geq 0\right\}$ be a branching process with offspring distribution $X^{(n)}$ and $X^{(n)}(0)=$ 1. Furthermore, write $Y^{(n)}(t)=\sum_{s=0}^{t} X^{(n)}(s)$ for the total progeny of the branching process at time $t$. Then, for even $t$, the number of individuals (not necessarily distinct) that have been chosen in the construction of the process $\mathcal{C}^{(n)}(t)$ has the same distribution as $Y^{(n)}(t / 2)$, and the number of groups (not necessarily distinct) that have been chosen is (stochastically) strictly smaller than $Y^{(n)}(t / 2)$.

We will now show that

$$
\mathrm{P}\left(Y^{(n)}(\lfloor\kappa \log n\rfloor) \geq n^{1 / 2-\varepsilon}\right)=o(1) \quad \text { as } n \rightarrow \infty
$$

for small enough $\varepsilon>0$, by Markov's inequality. Define $\mu_{n}:=\mathrm{E}\left[X^{(n)}\right]=\gamma^{2} m / n$ and note that $\beta \gamma^{2}(1-1 / \beta n) \leq \gamma^{2}\lfloor\beta n\rfloor / n=\gamma^{2} m / n \leq \beta \gamma^{2}$, so that $\mu_{n} \leq n$ and $\mu_{n} \rightarrow \mu:=\beta \gamma^{2}$. We have

$$
\mathrm{E}\left[Y^{(n)}(t)\right]=\mathrm{E}\left[\sum_{s=0}^{t} X^{(n)}(s)\right]=\sum_{s=0}^{t} \mu_{n}^{s} \leq \begin{cases}\frac{1}{1-\mu_{n}}, & \mu_{n}<1, \\ 1+t \mu_{n}^{t}, & \mu_{n} \geq 1 .\end{cases}
$$

For $\mu \leq 1$, we may take $0<\varepsilon<\frac{1}{2}$ :

$$
\mathrm{P}\left(Y^{(n)}\left((\lfloor\kappa \log n\rfloor) \geq n^{1 / 2-\varepsilon}\right)\right) \leq n^{-1 / 2+\varepsilon} O(\log n)=o(1) .
$$


For $\mu>1$, we take $0<\varepsilon<\frac{1}{2}-\kappa \log \mu$, so that $\varepsilon<\frac{1}{2}$ and $\frac{1}{2}-\kappa \log \mu-\varepsilon>0$ :

$$
\begin{aligned}
\mathrm{P}\left(Y^{(n)}(\lfloor\kappa \log n\rfloor) \geq n^{1 / 2-\varepsilon}\right) & \leq n^{-1 / 2+\varepsilon}\left(1+\lfloor\kappa \log n\rfloor \mu_{n}^{\lfloor\kappa \log n\rfloor}\right) \\
& \leq o(1)+\lfloor\kappa \log n\rfloor n^{-1 / 2+\varepsilon} \mu^{\kappa \log n} \\
& =o(1)+\lfloor\kappa \log n\rfloor n^{-1 / 2+\varepsilon} n^{\kappa \log \mu} \\
& =o(1)+\lfloor\kappa \log n\rfloor n^{-(1 / 2-\kappa \log \mu-\varepsilon)} \\
& =o(1) .
\end{aligned}
$$

Now let $A_{n}$ denote the event that all individuals and groups that have been chosen in the construction of $\mathcal{C}^{(n)}(t)$ are distinct, and let $B_{n}$ denote the event that $\left\{Y^{(n)}(\lfloor\kappa \log n\rfloor) \geq n^{1 / 2-\varepsilon}\right\}$. On $A_{n}$, clearly the subgraph of $\mathscr{B}^{(n)}(t)$ induced by $\mathfrak{C}^{(n)}(t)$ is a tree, that is, $A_{n} \subset T_{n}$. Hence,

$$
\begin{aligned}
\mathrm{P}\left(T_{n}\right) & \geq \mathrm{P}\left(A_{n}\right) \\
& =\mathrm{P}\left(A_{n} \mid B_{n}\right) \mathrm{P}\left(B_{n}\right)+\mathrm{P}\left(A_{n} \mid B_{n}^{\mathrm{c}}\right) \mathrm{P}\left(B_{n}^{\mathrm{c}}\right) \\
& \geq(1+o(1)) \mathrm{P}\left(A_{n} \mid B_{n}\right) \\
& \geq(1+o(1)) \prod_{k=1}^{n^{1 / 2-\varepsilon}}\left(1-\frac{k}{n}\right)\left(1-\frac{k}{m}\right) \\
& =(1+o(1)) \exp \left(\sum_{k=1}^{n^{1 / 2-\varepsilon}}\left(\log \left(1-\frac{k}{n}\right)+\log \left(1-\frac{k}{m}\right)\right)\right) \\
& =(1+o(1)) \exp \left(-\left(1+\frac{1}{\beta}\right) \sum_{k=1}^{n^{1 / 2-\varepsilon}}\left(\frac{k}{n}+o\left(\frac{k^{2}}{n^{2}}\right)\right)\right) \\
& =(1+o(1)) \exp (o(1)) \\
& =1+o(1),
\end{aligned}
$$

and the lemma is proved.

Proof of Theorem 3.1. The idea of the proof is to construct a branching process $\left\{Z^{(n)}(t)\right.$ : $t \geq 0$, with $Z^{(n)}(0)=1$, that counts the number of individuals infected by the epidemic in its initial stage, though not necessarily in chronological order. The branching process will be defined in such a way that the final size of the epidemic and the total progeny of the branching process are equal with high probability. As $n \rightarrow \infty$, we will have $Z^{(n)} \rightarrow Z$ in distributionwhere $Z$ is the branching process in the formulation of the theorem-and the theorem thus follows.

First, we describe the initial spread of the disease among the individuals/groups in the set $\mathcal{C}^{(n)}(\lfloor\kappa \log n\rfloor)$ with a process $\left\{\mathcal{E}^{(n)}(t): 0 \leq t \leq\lfloor\kappa \log n\rfloor\right\}$.

We only consider what happens on the set $T_{n}$, since the probability of the complementary set tends to 0 by Lemma 3.1. Furthermore, our construction will be such that $\mathcal{E}^{(n)}(t) \subseteq \mathcal{C}^{(n)}(t)$ for all $t$, implying that the nodes of $\mathcal{E}^{(n)}(\lfloor\kappa \log n\rfloor)$ themselves constitute a tree if seen as a subgraph of $\mathscr{B}^{(n)}$.

The construction of $\mathcal{E}^{(n)}(t)$ is similar to the construction of $\mathcal{C}^{(n)}(t)$ described in Lemma 3.1. Namely, we will define a sequence $\left\{\mathcal{F}^{(n)}(t): 0 \leq t \leq\lfloor\kappa \log n\rfloor\right\}$ and then set

$$
\mathcal{E}^{(n)}(t)=\bigcup_{0 \leq s \leq t} \mathcal{F}^{(n)}(s) .
$$


To this end, first let $\mathcal{F}^{(n)}(0)$ consist of the initial infective, that is, $\mathcal{F}^{(n)}(0)=\left\{v_{1}\right\}$. Then, for odd $t$, let $\mathcal{F}^{(n)}(t)$ consist of the groups of the individuals in $\mathcal{F}^{(n)}(t-1)$ that are at distance $t$ from $v_{1}$, that is,

$$
\mathcal{F}^{(n)}(t)=\left\{a \in \mathscr{D}^{(n)}(t) \text { : there exists } v \in \mathcal{F}^{(n)}(t-1) \text { such that } v \in a\right\}
$$

To define $\mathcal{F}^{(n)}(t)$ for even $t$, recall the percolation representation of the set of ultimately infected individuals in $g^{(n)}$ described before Theorem 3.1.

For a group $a$, let $v \stackrel{a}{\leftrightarrow} w$ denote that the vertices $v$ and $w$ belong to $a$, and that there exists a path of open edges - that is, edges that can be used for disease transmission—connecting $v$ and $w$, with the additional property that the whole path is contained in group a. Furthermore, let $\mathcal{K}_{a, v}=\{w \in a: v \stackrel{a}{\leftrightarrow} w\}$. This is to be thought of as the local outbreak in group $a$ caused by individual $v$, if $v$ itself becomes infected from outside of group $a$, excluding $v$ itself. As pointed out before the formulation of Theorem 3.1, given that $|a \backslash\{v\}|=k$, we have $\left|\mathcal{K}_{a, v}\right| \sim F_{k}$, where $F_{k}$ is the distribution of the final size of a homogeneous Reed-Frost epidemic initiated by a single infectious individual in a population with $k$ susceptible individuals (excluding the index case). Note that $|a \backslash\{v\}| \sim \operatorname{binomial}(n-1, \gamma / n)$, and, for future use, let $R^{(n)}$ be a random variable with distribution $\sum_{k} F_{k} \mathrm{P}(|a \backslash\{v\}|=k)$, that is, the size of a local outbreak in a group, not conditioning on the group size. Now, for even $t$, define $\mathcal{F}^{(n)}(t)$ to be the individuals infected in the local outbreaks caused by the individuals in $\mathcal{F}^{(n)}(t-2)$, that is,

$$
\mathcal{F}^{(n)}(t)=\left\{w \in \mathcal{K}_{a, v}: a \in \mathcal{F}^{(n)}(t-1), v \in \mathcal{F}^{(n)}(t-2)\right\}
$$

We will now study the growth of $\left|\mathcal{E}^{(n)}(t)\right|$. To this end, for $0 \leq t \leq \frac{1}{2}\lfloor\kappa \log n\rfloor$, define $Z^{(n)}(t)=$ $\left|\mathcal{F}^{(n)}(2 t)\right|$. Then, since the subgraph of $\mathscr{B}^{(n)}$ induced by $\mathcal{C}^{(n)}(2 t)$ is a tree for $t \leq \frac{1}{2}\lfloor\kappa \log n\rfloor$, by construction, $Z^{(n)}(t)$ is a branching process with a compound binomial offspring distribution. The generating function of the offspring distribution is

$$
f_{n}(s)=\mathrm{E}\left[s^{Z^{(n)}(1)}\right]=\left(1-\frac{\gamma}{n}+\frac{\gamma}{n} \mathrm{E}\left[s^{R^{(n)}}\right]\right)^{m} .
$$

For $t \geq \frac{1}{2}\lfloor\kappa \log n\rfloor$, we let $Z^{(n)}(t)$ evolve by the same branching mechanism, that is, as a discrete-time branching process with offspring distribution defined by (3.3). For $t \geq \frac{1}{2}\lfloor\kappa \log n\rfloor$, however, $Z^{(n)}(t)$ is no longer related to the epidemic process.

Let $Z_{\mathrm{tot}}^{(n)}=\sum_{t=0}^{\infty} Z^{(n)}(t)$. Since $Z^{(n)} \rightarrow Z$ in distribution, clearly $Z_{\mathrm{tot}}^{(n)} \rightarrow E$ in distribution. Let $\varepsilon>0$, let $k \in \mathbb{N}$, and take $n$ large enough so that

(i) $\mathrm{P}\left(T_{n}\right)>1-\varepsilon / 2$,

(ii) $k \leq \frac{1}{2}\lfloor\kappa \log n\rfloor$,

(iii) $\left|\mathrm{P}\left(Z_{\text {tot }}^{(n)} \leq k\right)-\mathrm{P}(E \leq k)\right| \leq \varepsilon / 2$. 
Note that if $k \leq \frac{1}{2}\lfloor\kappa \log n\rfloor$ then $\left\{E^{(n)} \leq k\right\} \cap T_{n}=\left\{Z_{\text {tot }}^{(n)} \leq k\right\} \cap T_{n}$. Hence,

$$
\begin{aligned}
\mathrm{P}\left(E^{(n)} \leq k\right) & =\mathrm{P}\left(E^{(n)} \leq k, T_{n}\right)+\mathrm{P}\left(E^{(n)} \leq k, T_{n}^{\mathrm{c}}\right) \\
& \leq \mathrm{P}\left(E^{(n)} \leq k, T_{n}\right)+\frac{\varepsilon}{2} \quad(\text { by (i) }) \\
& =\mathrm{P}\left(Z_{\text {tot }}^{(n)} \leq k, T_{n}\right)+\frac{\varepsilon}{2} \quad(\text { by (ii) }) \\
& \leq \mathrm{P}\left(Z_{\text {tot }}^{(n)} \leq k\right)+\frac{\varepsilon}{2} \\
& \leq \mathrm{P}(E \leq k)+\varepsilon \quad(\text { by (iii) }) .
\end{aligned}
$$

By (i), it follows that

$$
\begin{aligned}
\mathrm{P}\left(Z_{\text {tot }}^{(n)} \leq k, T_{n}\right) & =\mathrm{P}\left(Z_{\text {tot }}^{(n)} \leq k\right)-\mathrm{P}\left(Z_{\text {tot }}^{(n)} \leq k, T_{n}^{\mathrm{c}}\right) \\
& \geq \mathrm{P}\left(Z_{\text {tot }}^{(n)} \leq k\right)-\frac{\varepsilon}{2}
\end{aligned}
$$

and, thus,

$$
\begin{aligned}
\mathrm{P}\left(E^{(n)} \leq k\right) & =\mathrm{P}\left(E^{(n)} \leq k, T_{n}\right)+\mathrm{P}\left(E^{(n)} \leq k, T_{n}^{\mathrm{c}}\right) \\
& \geq \mathrm{P}\left(E^{(n)} \leq k, T_{n}\right) \\
& =\mathrm{P}\left(Z_{\text {tot }}^{(n)} \leq k, T_{n}\right) \quad(\text { by (ii) }) \\
& \geq \mathrm{P}\left(Z_{\text {tot }}^{(n)} \leq k\right)-\frac{\varepsilon}{2} \quad(\text { by (3.4)) } \\
& \geq \mathrm{P}(E \leq k)-\varepsilon \quad \text { (by (iii) }) .
\end{aligned}
$$

We conclude that $\left|\mathrm{P}\left(E^{(n)} \leq k\right)-\mathrm{P}(E \leq k)\right| \leq \varepsilon$. Since $\varepsilon>0$ and $k \in \mathbb{N}$ are arbitrary, this means that $E^{(n)} \rightarrow E$ in distribution, and the theorem is proved.

\section{The final outcome of the epidemic}

The branching process approach of the previous section basically gives no information about the behavior of the epidemic in the case of explosion. In this section we will elaborate a bit on this problem.

As already described, one way of getting a grip of the final outcome of the epidemic is to consider an edge percolation process on the underlying graph, where each edge in the graph is independently removed with probability $1-p$ and kept with probability $p$. The vertices that belong to the component of the initial infective in the graph so obtained correspond to the individuals that have experienced the infection at the end of the epidemic. If the structure of the thinned graph is known then this observation might be useful in investigating the final size of the epidemic. For instance, if there is a unique giant component in the thinned graph-that is, if the outcome of the percolation process contains a unique cluster of order $n$-then the relative size of this component gives the probability of an outbreak of order $n$ in the epidemic. Such an outbreak is often referred to as a major outbreak, and, in most epidemic models, the probability of such an outbreak coincides with the probability of explosion in the branching process describing the initial stages of the epidemic (denoted by $\pi$ in this paper). This however requires additional arguments. 
In our case the social network is a random intersection graph with $\alpha=1$. Unfortunately, to date there are no rigorous results concerning the component structure in a random intersection graph with $\alpha=1$, but see Behrisch (2007) for results when $\alpha \neq 1$. Also, in Newman (2003a), (implicit) expressions for the size of the largest component in a random graph construction which is similar to the random intersection graph are derived by heuristic means and it is observed that the relative final size of the giant component seems to decrease as the clustering in the graph increases. An argument in support of the claim that high clustering in a graph causes the components to be small is the following: consider an arbitrary graph with $n$ vertices and $k=O(n)$ edges, and assume that the clustering equals 1 . This implies that all subgraphs are complete. Hence, with $n_{\max }$ denoting the size of the largest subgraph, we find that the number of edges in the maximal subgraph is $\left(\begin{array}{c}n_{\max } \\ 2\end{array}\right)$. It follows that $n_{\max } \leq O(\sqrt{k})=O(\sqrt{n})$, that is, the relative size of the largest component tends to 0 .

Indeed, the lack of rigorous results concerning the components in a random intersection graph with $\alpha=1$ makes it harder to study the final size of an epidemic on such a graph. A second complicating circumstance is that thinning a random intersection graph gives rise to a graph that no longer belongs to the class of random intersection graphs; see Proposition 4.1, below. This means that, even if there were results for the component structure, these would not be applicable to a thinned graph. Hence, it remains an open problem to quantify the final outcome of the epidemic.

Proposition 4.1. Let $\Theta_{p}\left(g_{\beta, \gamma}^{(n)}\right)$ denote the graph generated by removing edges in $g_{\beta, \gamma}^{(n)}$ independently with probability $1-p$. There do not exist $\beta^{\prime}=\beta^{\prime}(\beta, \gamma, p)$ and $\gamma^{\prime}=\gamma^{\prime}(\beta, \gamma, p)$

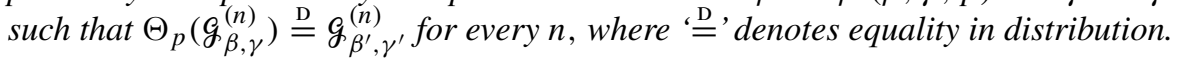

Proof. The idea of the proof is to observe that certain types of subgraphs will appear with different frequency in $\Theta_{p}\left(g_{\beta, \gamma}^{(n)}\right)$ as compared to $g_{\beta, \gamma}^{(n)}$. The subgraph that we will consider consists of four vertices and five edges, as shown below.

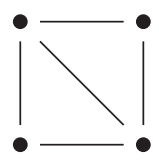

Write $K_{4}^{\prime}$ for this graph type, and note that it can be obtained, for instance, by removing one edge from a complete subgraph with four vertices, a graph type that we denote by $K_{4}$. Furthermore, we introduce the term vertex-induced subgraph for a subgraph of some given graph such that the subgraph consists of a subset of the vertices in the original graph together with all edges between these vertices that are present in the original graph.

The number $X_{4}$ of vertex-induced subgraphs of type $K_{4}$ in the random intersection graph $g_{\beta, \gamma}^{(n)}$ dominates the number of groups of size four in the construction of the random intersection graph. Since the size of a fixed group is $\operatorname{binomial}(n, \gamma / n)$ distributed, the number of groups of size four is binomial $\left(\lfloor\beta n\rfloor,\left(\begin{array}{c}n \\ 4\end{array}\right)(\gamma / n)^{4}(1-\gamma / n)^{n-4}\right)$ distributed and, hence, $\mathrm{E}\left[X_{4}\right] \geq O(n)$. It follows that the number $X_{4}^{\prime}(p)$ of vertex-induced subgraphs of type $K_{4}^{\prime}$ in the thinned graph $\Theta_{p}\left(g_{\beta, \gamma}^{(n)}\right)$ is also at least of the order $n$, since, as mentioned, one way of obtaining graphs of type $K_{4}^{\prime}$ is to remove one edge in graphs of type $K_{4}$, that is, $\mathrm{E}\left[X_{4}^{\prime}(p)\right] \geq(1-p) p^{5} \mathrm{E}\left[X_{4}\right] \geq O(n)$.

Now consider the number $X_{4}^{\prime}$ of vertex-induced subgraphs of type $K_{4}^{\prime}$ in the random intersection graph $g_{\beta, \gamma}^{(n)}$. This number is related to the number of ways that four individuals $v_{1}, \ldots, v_{4}$ can be assigned to different groups so that a graph of type $K_{4}^{\prime}$ is obtained. Consider, 
for instance, the following graph of type $K_{4}^{\prime}$.

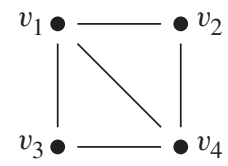

Write $\left\{\left(v_{i_{1}}, v_{i_{2}}\right)\left(v_{j_{1}}, v_{j_{2}}\right)\left(v_{k_{1}}, v_{k_{2}}\right)\right\}$ for the event that $v_{i_{1}}$ and $v_{i_{2}}$ share a group, that $v_{j_{1}}$ and $v_{j_{2}}$ share another group, and that $v_{k_{1}}$ and $v_{k_{2}}$ share yet another group. Then, for the above graph to arise, the individuals $v_{2}$ and $v_{3}$ cannot share any group - the probability that they avoid doing so goes to 1 as $n \rightarrow \infty$-and, in addition, one of the following events must occur:

$$
\begin{gathered}
\left\{\left(v_{1}, v_{2}, v_{4}\right)\left(v_{1}, v_{3}, v_{4}\right)\right\}, \\
\left\{\left(v_{1}, v_{2}, v_{4}\right)\left(v_{1}, v_{3}\right)\left(v_{3}, v_{4}\right)\right\}, \\
\left\{\left(v_{1}, v_{3}, v_{4}\right)\left(v_{1}, v_{2}\right)\left(v_{2}, v_{4}\right)\right\}, \\
\left\{\left(v_{1}, v_{2}\right)\left(v_{1}, v_{4}\right)\left(v_{2}, v_{4}\right)\left(v_{1}, v_{3}\right)\left(v_{3}, v_{4}\right)\right\} .
\end{gathered}
$$

It follows that

$$
\begin{aligned}
\mathrm{E}\left[X_{4}^{\prime}\right] & \leq n^{4}\left(\begin{array}{c}
\lfloor\beta n\rfloor \\
2
\end{array}\right)\left(\frac{\gamma}{n}\right)^{6}+2 n^{4}\left(\begin{array}{c}
\lfloor\beta n\rfloor \\
3
\end{array}\right)\left(\frac{\gamma}{n}\right)^{7}+n^{4}\left(\begin{array}{c}
\lfloor\beta n\rfloor \\
5
\end{array}\right)\left(\frac{\gamma}{n}\right)^{10} \\
& =O(1) .
\end{aligned}
$$

The number of vertex-induced subgraphs of type $K_{4}^{\prime}$ in a random intersection graph is hence $O(1)$, while, in a thinned random intersection graph, it is of order $n$. This proves the proposition.

\section{Numerical results}

In this section we numerically investigate the epidemic threshold $R_{0}$ and the probability $\pi$ of explosion in the epidemic-recall Corollary 3.1. We have $R_{0}=\beta \gamma \mathrm{E}[R]$, where the distribution of $R$ is specified in (3.1), and $\pi:=1-\rho$, where $\rho$ is the smallest nonnegative root of the equation $f(\rho)=\rho$ and $f$ is the generating function specified in (3.2). Using the recursive formulae for the distribution $F_{k}$ of the final size of a Reed-Frost epidemic in a homogeneous population of size $k$-see, e.g. Andersson and Britton (2000, Section 1.2)—numerical values of $R_{0}$ and $\pi$ are easily obtained for fixed values of $\beta$ and $\gamma$ (this requires some truncations that do not affect the results).

We are particularly interested in how $\rho$ and $R_{0}$ are affected when the (asymptotic) clustering $c=(1+\beta \gamma)^{-1}$ is varied, and, to be able to compare results for different values of $c$, the mean degree $\mu=\beta \gamma^{2}$ in the graph is kept fixed. In Figure 1 the parameters $R_{0}$ and $\pi$ are plotted against $c$ for three different values of the infection probability $p$. Let us comment a bit on these plots.

First we investigate the value of $R_{0}$ in the limit as $c \rightarrow 0$. Since $\mu$ is fixed, we see that $c=(1+\mu / \gamma)^{-1} \rightarrow 0$ implies that $\gamma \rightarrow 0$ as well. Asymptotically, the degree distribution in our random intersection graph is compound Poisson with generating function

$$
g(s)=\exp \left(\beta \gamma\left(\mathrm{e}^{\gamma(s-1)}-1\right)\right),
$$

which converges to $\mathrm{e}^{\mu(s-1)}$ as $\gamma \rightarrow 0$. The limiting degree distribution as $c \rightarrow 0$ is hence Poisson $(\mu)$ and, after thinning the graph, removing each edge independently with probability $1-p$, the degrees are Poisson distributed with mean $p \mu$. Since the graph obtained by such 

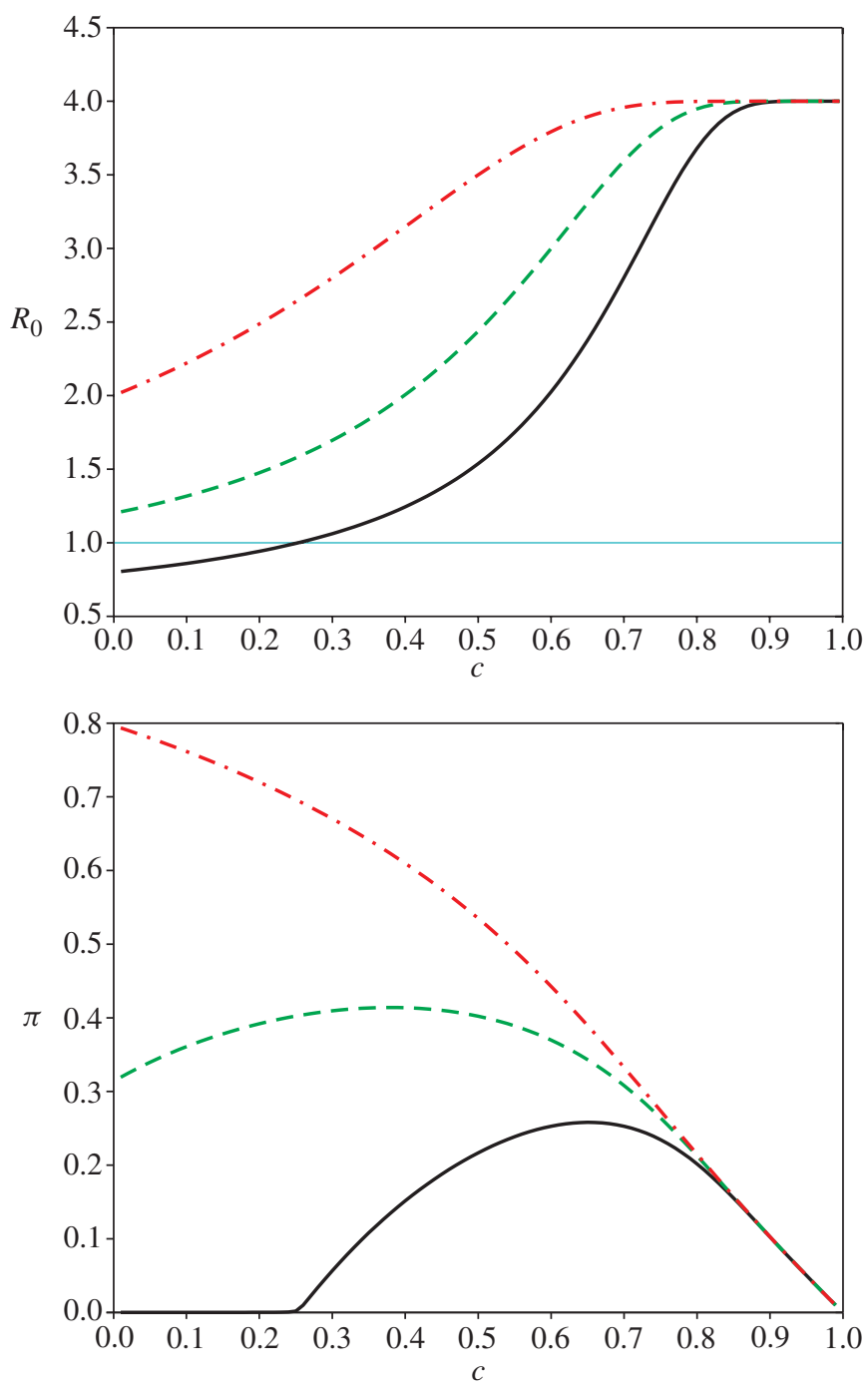

FIGURE 1: In the top plot, $R_{0}$ is plotted against $c$ for fixed $\mu=4$ and for three choices of $p: p=0.2$ (solid line), $p=0.3$ (dashed line), and $p=0.5$ (dash-dot line). The bottom plot shows how the probability $\pi$ of explosion in the epidemic varies with $c$ for fixed $\mu=4$ and the same choices of $p$ as above.

a thinning can be thought of as representing the outcome of the epidemic, it is reasonable to suspect that $R_{0}=p \mu$ in the limit as $c \rightarrow 0$. Indeed, it can be seen in the top plot of Figure 1 that $R_{0} \rightarrow p \mu$ as $c \rightarrow 0$.

In the top plot of Figure 1 it can also be seen that $R_{0}$ increases with $c$, that is, higher clustering makes it easier for epidemics to take off. This is in line with the findings in Newman (2003a). Let us give a heuristic explanation of why this should be the case: first note that, since the mean degree $\mu$ in the graph is fixed, an increase in $c=(1+\mu / \gamma)^{-1}$ is equivalent to an increase in $\gamma$ and a decrease in $\beta$ of the order $\gamma^{-2}$. Also, recall that the mean number of groups that an individual is a member of is $\beta \gamma$ and the mean group size is $\gamma$. Hence, increased clustering with fixed mean degree means that individuals are members of fewer but larger groups. Combining 
this with the observation that the probability for an individual to avoid infection from some index case with whom he/she shares a group decreases geometrically with the group size, it follows that it should be easier for an epidemic to take off when the clustering is large. In fact, we have $R_{0} \rightarrow \mu$ as $c \rightarrow 1$, that is, in the limit of large clustering, the infection probability $p$ does not matter (as long as it is positive) for the value of $R_{0}$.

The bottom plot in Figure 1 shows how the probability $\pi$ of explosion in the epidemic varies with $c$. For instance, it can be seen that $\pi \rightarrow 0$ as $c \rightarrow 1$. In Section 4 we argued that the relative size of the largest component in a graph with maximal clustering is 0 in the limit of large graph size. If the probability of explosion in the epidemic coincides with the relative size of the largest component in the graph representing the outcome of the epidemic then indeed it follows from this that $\pi \rightarrow 0$ as $c \rightarrow 1$. Furthermore, it is interesting to note that the decrease of $\pi$ towards 0 is not monotone for all values of $p$. Clearly, if a low value of $c$ prevents explosion, while explosion is possible for a larger value of $c$ - this is the case, for instance, for $p=0.2$ - then we will see an increase in $\pi$ from 0 to a positive value when the threshold is passed. But, as the curve for $p=0.3$ reveals, even if $\pi$ is already positive at $c=0$, it can be the case that it increases with $c$ in some interval before it starts to decrease.

\section{Discussion}

In the present paper we have analyzed how the clustering in a random network affects how an infectious disease propagates in the network, assuming that the size of the network is large. In particular, using a random intersection graph construction, we have rigorously derived the limiting probability of an explosion in the epidemic and a threshold parameter indicating if this probability is 0 or positive.

The motivation for analyzing an epidemic on a random network with positive clustering is of course that most empirical social networks manifest positive clustering, so predictions based on epidemic models neglecting such clustering, i.e. most epidemic models, must be interpreted with caution. There are of course several other features in empirical networks, not considered in the present paper, which should also be taken into account for predictions to be reliable. One such feature is the degree distribution, which in many social networks has been observed to follow a power-law distribution. The graph model used in this paper gives compound Poisson distributions for the degrees, but the model is generalized in Deijfen and Kets (2007) to allow for power-law degree distributions. It would be interesting to study how an epidemic on such a generalized graph is affected by the exponent in the power law. Another feature that has been observed in many social networks is positive degree correlation, that is, individuals with high or low degrees tend to be connected to other individuals with high or, respectively, low degrees. Because of the group structure, this is likely to be the case in a random intersection graph, but it remains to quantify the correlation.

A possible generalization of the studied model would be to distinguish between different types of individuals, and to assume that both network characteristics as well as transmission probabilities depend on the type of an individual; see, e.g. Ball and Clancy (1993). Another extension, motivated by real-world epidemics, is to leave the Reed-Frost paradigm, in which the events that different neighbors of a given infective becomes infected are independent. If, for example, the infectious period is taken to be random, then these events are positively correlated; see, e.g. Andersson and Britton (2000, Chapter 2). Unfortunately, by relaxing the independence assumption, the analysis of the model becomes much more complicated. 
Perhaps the most obvious continuation of the present work is however to derive fully rigorous results about the final size of the epidemic in the case of explosion. The (relative) final size of the epidemic then most likely coincides with the probability of explosion, a quantity derived in the present paper, but a proof of this is still missing.

\section{Acknowledgement}

We thank an anonymous referee for insightful comments and suggestions which helped us clarify and correct parts of the proofs.

\section{References}

Andersson, H. (1998). Limit theorems for a random graph epidemic model. Ann. Appl. Prob. 8, 1331-1349.

Andersson, H. (1999). Epidemic models and social networks. Math. Scientist 24, 128-147.

Andersson, H. And Britton, T. (2000). Epidemic Models and Their Statistical Analysis. Springer, Berlin.

Athreya, K. B. ANd Ney, P. E. (1972). Branching Processes. Springer, New York.

Ball, F. AND ClanCY, D. (1993). The final size and severity of a generalized stochastic multi-type epidemic model. Adv. Appl. Prob. 25, 721-736.

Ball, F., Mollison, D. and Scalia-Tomba, G. (1997). Epidemics with two levels of mixing. Ann. Appl. Prob. 7, 46-89.

Behrisch, M. (2007). Component evolution in random intersection graphs. Electron. J. Combinatorics 14, 12 pp.

DeiJfen, M. AND Kets, W. (2007). Random intersection graphs with tunable degree distribution and clustering. Submitted.

Dorogovtsev, S. And Mendes, J. (2003). Evolution of Networks. From Biological Nets to the Internet and WWW. Oxford University Press.

Durrett, R. (2006). Random Graph Dynamics. Cambridge University Press.

Fill, J., Scheinerman, E. AND Singer-Cohen, K. (2000). Random intersection graphs when $m=\omega(n)$ : an equivalence theorem relating the evolution of the $G(n, m, p)$ and $G(n, p)$ models. Random Structures Algorithms 16, 156-176.

GODEHARDT, E. AND JAWORSKI, J. (2002). Two models of random intersection graphs for classification. In Exploratory Data Analysis in Empirical Research, eds M. Schwaiger and O. Opitz, Springer, Berlin, pp. 67-81.

Karoński, M., Scheinerman, E. ANd Singer-Cohen, K. (1999). On random intersection graphs: the subgraphs problem. Combinatorics Prob. Comput. 8, 131-159.

Neal, P. (2004). SIR epidemics on Bernoulli graphs. J. Appl. Prob. 40, 779-782.

NeAL, P. (2006). Multitype randomized Reed-Frost epidemics and epidemics upon graphs. Ann. Appl. Prob. 16, 11661189.

Newman, M. (2003a). Properties of highly clustered networks. Phys. Rev. E 68, 026121.

Newman, M. (2003b). The structure and function of complex networks. SIAM Rev. 45, 167-256.

Newman, M., Barabási, A. And Watts, D. (2006). The Structure and Dynamics of Networks. Princeton University Press.

SINGER, K. (1995). Random intersection graphs. Doctoral Thesis, Johns Hopkins University.

StaRk, D. (2004). The vertex degree distribution of random intersection graphs. Random Structures Algorithms 24, 249-258.

Trapman, P. (2007). On analytical approaches to epidemics on networks. Theoret. Pop. Biol. 71, 160-173.

Von Bahr, B. And Martin-Löf, A. (1980). Threshold limit theorems for some epidemic processes. Adv. Appl. Prob. 12, 319-349. 